\title{
Magnetocaloric effect in itinerant electron metamagnetic systems $\mathrm{La}\left(\mathrm{Fe}_{1-x} \mathrm{Co}_{x}\right)_{11.9} \mathrm{Si}_{1.1}$
}

\author{
F. X. Hu ${ }^{a)}$ \\ Department of Physics, Capital Normal University, Beijing 100037, People's Republic of Chinaand \\ Department of Physics, The University of Hong Kong, Pokfulam Road, Hong Kong \\ J. Gao \\ Department of Physics, The University of Hong Kong, Pokfulam Road, Hong Kong, China \\ X. L. Qian \\ Department of Physics, Capital Normal University, Beijing 100037, People's Republic of China \\ Max llyn and A. M. Tishin \\ Department of Physics, M.V. Lomonosov Moscow State University, Moscow, 119899, Russia
}

J. R. Sun and B. G. Shen

Beijing National Laboratory for Condensed Matter Physics, Institute of Physics, Chinese Academy of Sciences, Beijing 100080, China

(Presented on 9 November 2004; published online 13 May 2005)

The $\mathrm{NaZn}_{13}$-type compounds $\mathrm{La}\left(\mathrm{Fe}_{1-x} \mathrm{Co}_{x}\right)_{11.9} \mathrm{Si}_{1.1} \quad(x=0.04,0.06,0.08)$ were successfully synthesized, in which the $\mathrm{Si}$ content is the limit that can be reached by arc-melting technique. $T_{C}$ is tunable from 243 to $301 \mathrm{~K}$ with Co doping from $x=0.04$ to 0.08 . Great magnetic entropy change $\Delta S$ in a wide temperature range from $\sim 230$ to $\sim 320 \mathrm{~K}$ has been observed. The adiabatic temperature change $\Delta T_{\text {ad }}$ upon changing magnetic field was also directly measured. $\Delta T_{\text {ad }}$ of sample $x=0.06$ reaches $\sim 2.4 \mathrm{~K}$ upon a field change from 0 to $1.1 \mathrm{~T}$. The temperature hysteresis upon phase transition is small, $\sim 1 \mathrm{~K}$, for all samples. The influence of Co doping on itinerant electron metamagnetic transition and magnetic entropy change is discussed. (c) 2005 American Institute of Physics. [DOI: 10.1063/1.1847071]

Magnetocaloric effect (MCE) has been a topic of intense research in the past decades. MCE is induced via the coupling of the magnetic sublattice with the magnetic field. It can be characterized by isothermal entropy change $\Delta S$, as well as adiabatic temperature change $\Delta T_{\text {ad. }}$. The compounds with cubic $\mathrm{NaZn}_{13}$-type structure have been recently suggested to be appropriate materials for exploring efficient magnetic refrigerants. ${ }^{1-4}$ The large magnetic entropy change reported in this class of ferromagnetic materials warrants further experimental and theoretical studies. The $\mathrm{NaZn}_{13}$-type compounds $\mathrm{LaFe}_{13-x} \mathrm{Si}_{x}$ with low $\mathrm{Si}$ content show an itinerant electron metamagnetic transition above Curie temperature $T_{C}$ and a negative lattice expansion at $T_{C}$. With decreasing $\mathrm{Si}$ content, the nature of the phase transition evolves from second-order to first-order and the first-order nature is strengthened by further reducing the Si content. We found that incorporation of Co weakens the first-order nature of the transition and drives $T_{C}$ to a higher temperature. A proper combination of $\mathrm{Si}$ and Co could make $T_{C}$ near room temperature and preserve the first-order nature of the transition, which is characterized by a sharp change of lattice parameter. In this paper, we successfully synthesized $\mathrm{NaZn}_{13}$-type compounds $\mathrm{La}\left(\mathrm{Fe}_{1-x} \mathrm{Co}_{x}\right)_{11.9} \mathrm{Si}_{1.1}(x=0.04,0.06,0.08)$, in which the $\mathrm{Si}$ content is the lowest limit that can be reached by arc-melting technique, and systematically investigated their

\footnotetext{
${ }^{a)}$ Author to whom correspondence should be addressed; electronic mail: fxhu2002@hku.hk
}

magnetocaloric effect. $T_{C}$ is tunable from 243 to $301 \mathrm{~K}$ with Co doping from $x=0.04$ to 0.08 . The influence of Co doping on itinerant electron metamagnetic transition and magnetic entropy change is discussed.

The samples employed in the present investigation were prepared by repeated arc-melting appropriate amounts of starting materials. The commercial purity of the starting materials $\mathrm{La}, \mathrm{Fe}, \mathrm{Co}$, and $\mathrm{Si}$ are 99.9 wt \%, 99.99 wt \%, 99.9 wt $\%$, and 99.999 wt \%, respectively. The ingots were wrapped with Ta foil individually and homogenized in a sealed quartz tube with a high vacuum of $10^{-4} \mathrm{~Pa}$ for 30 days at $1323 \mathrm{~K}$, then quenched in liquid nitrogen. Quenching is important to obtain a stable compound with low Si content for this class of alloys. The $\mathrm{Si}$ composition in present samples $\mathrm{La}\left(\mathrm{Fe}_{1-x} \mathrm{Co}_{x}\right)_{11.9} \mathrm{Si}_{1.1}(x=0.04,0.06,0.08)$ is the limit that can be reached by using such a synthesizing method. Further reducing Si content would cause appearance of a large amount of $\alpha$-Fe. X-ray diffraction (XRD) analysis show that the present samples crystallized in a single phase of cubic NaZn13-type structure. A minor $\alpha$-Fe impurity was observed, the amount of which is around 4-6 wt \% estimated from Rietveld refinement of the XRD data, as well as the chemical analysis by inductively coupled plasma-atomic emission spectrometry (ICP-AES). All magnetic measurements were performed using a superconducting quantum interference device (SQUID) magnetometer. The Curie temperature $T_{C}$ was determined to be 243,274 , and $301 \mathrm{~K}$ for samples $x=0.04,0.06,0.08$ from the temperature dependent 


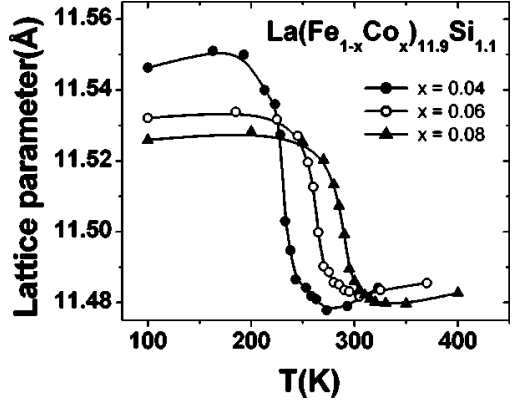

FIG. 1. The temperature dependent lattice parameter of $\mathrm{La}\left(\mathrm{Fe}_{1-x} \mathrm{Co}_{x}\right)_{11.9} \mathrm{Si}_{1.1}$ $(x=0.04,0.06,0.08)$ compounds.

magnetization measured under a field of $0.01 \mathrm{~T}$. The direct measurement of magnetocaloric effect was carried out under adiabatic conditions with a continuous registration of a temperature change upon a fast increase of applied magnetic field. The rate of the field change is about $0.7 \mathrm{~T} / \mathrm{s}$. The accuracy of the measurements is about $5 \%$.

In order to study the structure change on altering magnetic state with temperature, XRD measurements at various temperatures were performed for all samples. It is found that the crystal structure remains cubic $\mathrm{NaZn}_{13}$-type, but the lattice parameter changes dramatically at $T_{C}$, implying a firstorder transition. Figure 1 shows the temperature dependence of lattice parameter obtained from the XRD spectra, noting the large negative thermal expansion near $T_{C}$. The lattice parameter in the ferromagnetic state, for different samples $x=0.04,0.06$, and 0.08 , is bigger than that in the paramagnetic state by $\sim 0.63 \%, 0.44 \%$, and $0.41 \%$, respectively, in the vicinity of $T_{C}$. The substitution of Co for Fe atoms influences the magnetovolume effect. The amount of lattice contraction gradually decreases with increasing Co doping. Careful measurements of ac susceptibility on heating and cooling indicate that temperature hystereses of the transitions for all samples are small, $\sim 1 \mathrm{~K}$. In the past, several theories were used to describe magneto-elastic effect in itinerant magnetic systems. ${ }^{5,6}$ A widely used theory is the localmoment volume magnetostriction, in which the magnetovolume effect arises from the volume dependence of the exchange integral between spins. A theory developed by applying the Stoner band model suggested that the magnetovolume effect is connected with the increase of the kinetic energy of the electron system due to the splitting of the $3 d$ band. For $\mathrm{LaFeAl}$ compounds with $\mathrm{NaZn}_{13}$-type structure, previous investigations ${ }^{5}$ indicated that the magnetovolume effect comes from both the local-moment and band part, but the latter contributes much more than the former. For understanding the origin of the large negative expansion near $T_{C}$ in present compounds, detailed information about the band structure is still needed.

Figure 2(a) displays the magnetization isotherms of $\mathrm{La}\left(\mathrm{Fe}_{1-x} \mathrm{Co}_{x}\right)_{11.9} \mathrm{Si}_{1.1}(x=0.04,0.06,0.08)$ measured on field increase in a wide temperature range with different temperature steps. In the vicinity of $T_{C}$, the temperature step of $2 \mathrm{~K}$ is chosen and a step of $5 \mathrm{~K}$ for the far regions. Figure 2(b) shows selected $M-H$ curves for increasing and decreasing fields. One can find that the magnetic hysteresis upon altering field is small for all samples. Moreover, the substitution

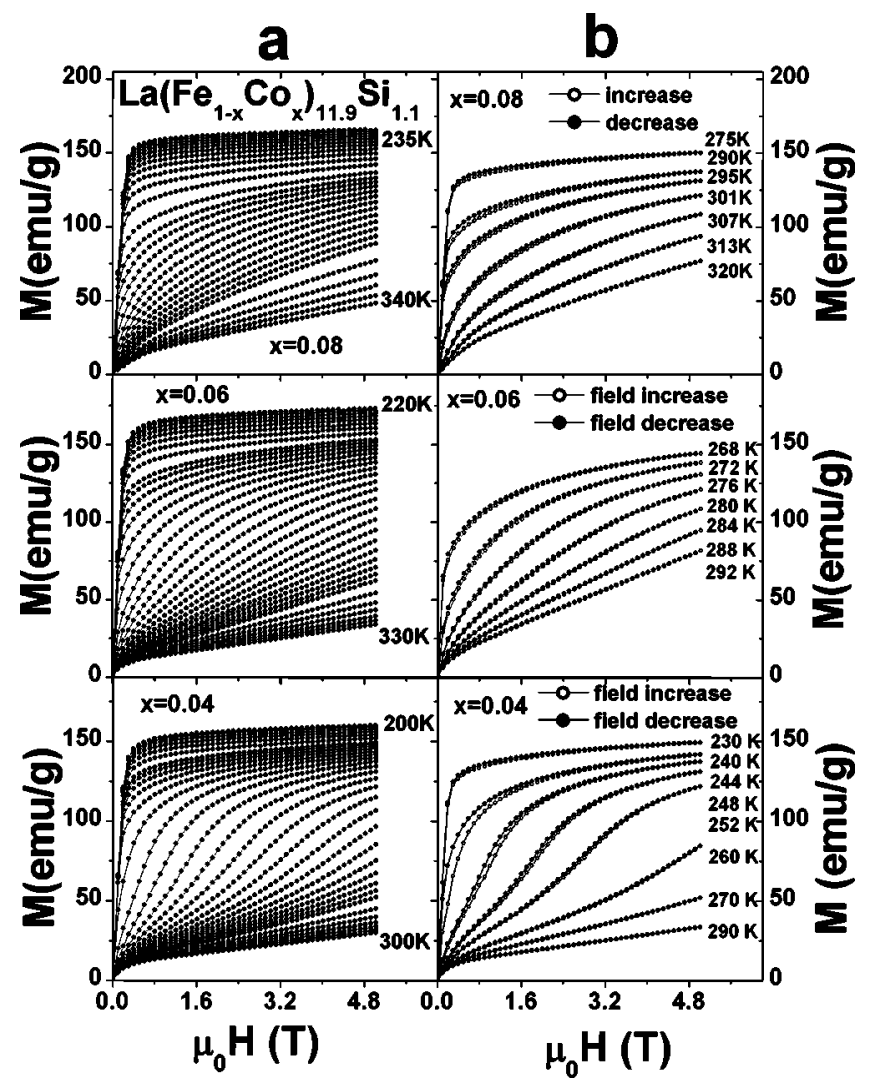

FIG. 2. Magnetization isotherms of $\mathrm{La}\left(\mathrm{Fe}_{1-x} \mathrm{Co}_{x}\right)_{11.9} \mathrm{Si}_{1.1}(x=0.04,0.06$, 0.08 ) compounds. (a) Isotherms on field increase. Temperature step is $2 \mathrm{~K}$ in the vicinity of $T_{C}$, and $5 \mathrm{~K}$ for the regions far away from $T_{C}$. (b) Selected isotherms measured on field increase and decrease.

of $\mathrm{Co}$ for $\mathrm{Fe}$ atoms further reduces the magnetic hysteresis. The small field hysteresis is considered to be a favorable characteristic for magnetic refrigeration applications.

Figure 3 shows the magnetic entropy change $\Delta S$ as functions of temperature and magnetic field obtained by using Maxwell relation $\Delta S(T, H)=\int_{0}^{H}(\partial M / \partial T)_{H} d H .^{1-4}$ The $\Delta S$ of $\mathrm{Gd}$ is also presented for comparison. One can find that the $\mathrm{La}\left(\mathrm{Fe}_{1-x} \mathrm{Co}_{x}\right)_{11.9} \mathrm{Si}_{1.1}$ compounds show large $\Delta S$ in a wide temperature range from $\sim 230$ to $\sim 320 \mathrm{~K}$. The $\Delta S$ significantly exceeds that of Gd. The $\Delta S$ magnitude decreases with increasing Co doping, which may correlate with the weakness of the magnetovolume effect (see Fig. 1). The weaken-

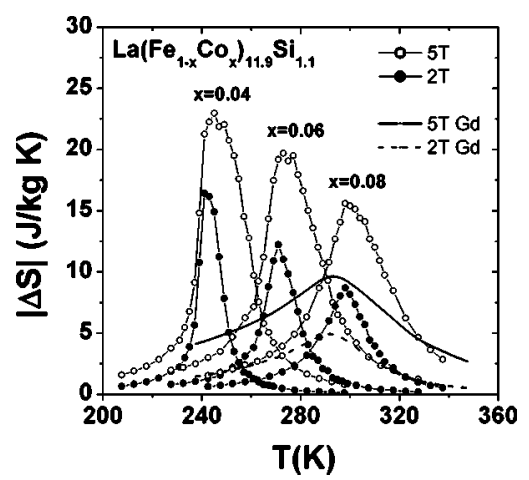

FIG. 3. Magnetic entropy change $|\Delta S|$ of $\mathrm{La}\left(\mathrm{Fe}_{1-x} \mathrm{Co}_{x}\right)_{11.9} \mathrm{Si}_{1.1} \quad(x=0.04$, $0.06,0.08)$ in comparison with that of $\mathrm{Gd}$ for the magnetic field changes from 0 to 2 and from 0 to $5 \mathrm{~T}$. 

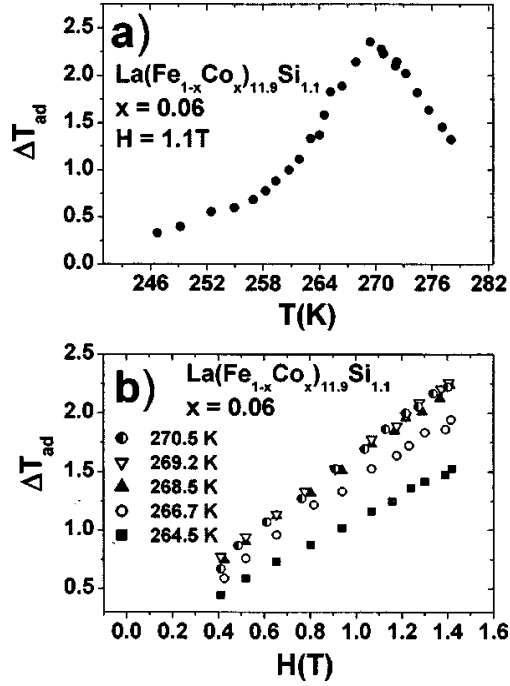

FIG. 4. (a) Adiabatic temperature change $\Delta T_{\text {ad }}$ of sample $x=0.06$ as a function of temperature obtained by direct measurements upon a field change from 0 to $1.1 \mathrm{~T}$. (b) The $\Delta T_{\text {ad }}$ as a function of applied magnetic field for different temperatures.

ing of magnetovolume effect would lead to a lessening of the slope of $M-T$ curves near $T_{C}$, and thus a decrease of $\Delta S$ according to Maxwell relation.

The asymmetrical broadening of $\Delta S$ peak with increasing field for $\mathrm{La}\left(\mathrm{Fe}_{1-x} \mathrm{Co}_{x}\right)_{11.9} \mathrm{Si}_{1.1}$ is also observed, which is a result of the field-induced itinerant-electron metamagnetic (IEM) transition from paramagnetic to ferromagnetic state above $T_{C}{ }^{1}$ Increasing Co content makes the phenomenon of asymmetrical broadening become weak. It seems that the substitution of the Co for Fe atoms can suppress the IEM transition in the present compounds. The phenomenon of IEM transition was historically observed in many $d$ electron systems. Yamada discussed it by taking into account the effect of spin fluctuations on the Ginzburg-Landau theory. The field-induced IEM transition is closely associated with the double minima of the paramagnetic and the ferromagnetic state in the magnetic-free energy as a function of magnetization. The applied fields drive the energy minimum of ferromagnetic state lower than that of paramagnetic state above $T_{C}$, resulting in the IEM transition. Generally, IEM transition is related to a special $d$ electron band structure which exhibits a sharp peak of the density of states (DOS) just below the Fermi level. ${ }^{7}$ It is known that the IEM transition in Laves phase compounds are attributed to unique Co $3 d$ band structure. ${ }^{7,8}$ Unfortunately, no information on the band structure is available for present compounds. Their IEM transition may be influenced by not only the electronic structure but also the elastic energy change. ${ }^{7-9}$ A reduction of the elastic energy change with Co doping (see Fig. 1) may be a possible reason of the gradual weakness of the IEM transition with increasing Co content.

In order to make sure the potential of present compounds as candidates for magnetic refrigerants, we chose sample $x$ $=0.06$ and directly measured the adiabatic temperature change $\Delta T_{\text {ad }}$ upon a change of magnetic field. Figure 4(a) displays the temperature dependent $\Delta T_{\text {ad }}$ collected on heat- ing the sample from $240 \mathrm{~K}$ to $280 \mathrm{~K}$ upon a field change from 0 to $1.1 \mathrm{~T}$. One can see the peak value of $\Delta T_{\text {ad }}$ reaches $2.4 \mathrm{~K}$. The peak position of $\Delta T_{\text {ad }}$ was found around $3 \mathrm{~K}$ less than $T_{C}$ defined by thermal magnetization curves. This difference in picking $\Delta T_{\text {ad }}$ temperature and $T_{C}$ may be explained by a simple thermodynamic model ${ }^{10}$ and could be accounted as quite reasonable. The field dependent $\Delta T_{\text {ad }}$ collected at different temperatures in the vicinity of $T_{C}$ is shown in Fig. 4(b). One can find that $\Delta T_{\text {ad }}$ collected at present temperatures starts to increase at the same magnetic field of $H$ $\sim 0.4 \mathrm{~T}$, and has a nearly linear dependence on applied field in the region of $0.4 \mathrm{~T}<H<1.4 \mathrm{~T}$. All $\Delta T_{\text {ad }}$ curves do not display a saturation behavior. It means that adiabatic temperature change would increase noticeably with increasing field.

In summary, $\mathrm{La}\left(\mathrm{Fe}_{1-x} \mathrm{Co}_{x}\right)_{11.9} \mathrm{Si}_{1.1} \quad(x=0.04,0.06,0.08)$ compounds with tunable $T_{C}$ from 243 to $301 \mathrm{~K}$ are successfully synthesized, in which the Si content reaches the lowest limit by using the present synthesis method. The magnetic ordering at $T_{C}$ is accompanied by a large increase of lattice parameter without changing the $\mathrm{NaZn}_{13}$-type structure. The transition is of first-order in nature with small temperature and field hystereses. Large magnetic entropy change in a wide temperature range from $\sim 230$ to $\sim 320 \mathrm{~K}$ is observed. The substitution of $\mathrm{Co}$ for $\mathrm{Fe}$ increases $T_{C}$ but reduces the amount of lattice contraction and suppresses the IEM transition gradually, thereby leads to a decrease of $\Delta S$ and weakens the asymmetrical broadening of the $\Delta S$ peak. The results of adiabatic temperature change obtained by direct measurements are also reported. Large values of the isothermal entropy change and adiabatic temperature change together with nonsaturated behavior of the field dependence of the MCE confirmed the large potential of present compounds as magnetic refrigerants in a wide temperature range near room temperature.

\section{ACKNOWLEDGMENTS}

This work has been supported by the Beijing Nature Science Foundation of China under Grant No. 1012002, the National Natural Science Foundation of China, CRCG of the University of Hong Kong, and Russian Foundation for Basic Research under Grant No. 04-02-16709-a.

${ }^{1}$ F. X. Hu, B. G. Shen, J. R. Sun, Z. H. Cheng, G. H. Rao, and X. X. Zhang, Appl. Phys. Lett. 78, 3675 (2001).

${ }^{2}$ F. X. Hu, B. G. Shen, J. R. Sun, and Z. H. Cheng, Phys. Rev. B 64, 012409 (2001)

${ }^{3}$ F. X. Hu, B. G. Shen, J. R. Sun, G. J. Wang, and Z. H. Cheng, Appl. Phys. Lett. 80, 826 (2002)

${ }^{4}$ A. Fujita, S. Fujieda, Y. Hasegawa, and K. Fukamichi, Phys. Rev. B 67, 104416 (2003)

${ }^{5}$ T. T. M. Palstra, G. J. Nieuwenhuys, J. A. Mydosh, and K. H. J. Buschow, Phys. Rev. B 31, 4622 (1985).

${ }^{6}$ P. Entel and M. Schroter, Physica B 161, 160 (1989).

${ }^{7}$ H. Yamada, Phys. Rev. B 47, 11211 (1993).

${ }^{8}$ H. Saito, T. Yokoyama, K. Fukamichi, K. Kamishima, and T. Goto, Phys. Rev. B 59, 8725 (1999).

${ }^{9}$ P. Mohn and K. Schwarz, J. Magn. Magn. Mater. 104, 685 (1992).

${ }^{10}$ V. K. Pecharsky, A. M. Tishin, and K. A. Gschneidner, Phys. Rev. B 59, 503 (1999) 